\title{
TINY MID- AND LONG-WAVE INFRARED SPECTROMETER MODULE WITH A MEMS DUAL-BAND FABRY-PÉROT FILTER
}

\author{
Martin Ebermann ${ }^{1}$, Marco Meinig ${ }^{2}$, Steffen Kurth ${ }^{2}$, Karla Hiller $^{3}$, Elvira Gittler ${ }^{4}$, Norbert Neumann ${ }^{1}$ \\ ${ }^{1}$ InfraTec GmbH, Gostritzer Strasse 61-63, 01217 Dresden, Germany \\ ${ }^{2}$ Fraunhofer Institute for Electronic Nano Systems, Technologie-Campus 3, 09126 Chemnitz, Germany \\ ${ }^{3}$ Chemnitz Univ. of Technology, Center for Microtechnologies, 09126 Chemnitz, Germany \\ ${ }^{4}$ Jenoptik Optical Systems GmbH, Göschwitzer Strasse 25, 07745 Jena, Germany
}

\begin{abstract}
This paper reports on new dual-band Fabry-Pérot-Filters (DB-FPF) and spectrometer modules, which can be operated simultaneously in the mid- and long-wave infrared by using the first and second interference order. With this design the restrictions of the usable tuning range of FP-filters, which are due to the limited free spectral range (FSR) and the bandwidth of the bragg reflectors, can be partly solved. In the LWIR $\mathrm{Ge}, \mathrm{ZnS}$ and a new low refractive dielectric material were used for the reflectors, giving additional degrees of freedom for the design. In addition the tensile and compressive stress of the optical thin films was compensated within the dielectric layer stack by a sophisticated design. First samples of dual-band reflectors, optimized for $4-5 \mu \mathrm{m}$ and $8-10.5 \mu \mathrm{m}$, were fabricated and tested in tunable FP-Filters. The reflectors exhibit a very low curvature and tilting, which gives rise to a high finesse and transmission of the filters. The DB spectrometer module is based on a dual channel pyroelectric detector. The first and second order is separated by means of a dichroic beamsplitter and two broad bandpass filters. Compared to conventional 50:50 beamsplitters this solution minimizes signal losses. The complete spectrometer module fits in a TO-8 package.
\end{abstract}

\section{Introduction}

Conventional IR analysis systems and spectrometers are complex and expensive instruments with very limited portability because of their size and power consumption. Micro-spectrometers on the other hand can be small, robust and less costly to manufacture. New application fields including medical diagnostics and detection of hazardous substances in industrial and security environments could be opened with the application of MEMS tunable infrared filters. Such filters for the $3-5 \mu \mathrm{m}$ MWIR range have been reported from several groups. ${ }^{[1]}{ }^{[2]}[3]$ Cost effective and highly miniaturized spectrometer modules for infrared absorption spectroscopy in the mid-wave infrared (MWIR) based on the hybrid integration of pyroelectric detectors and MEMS Fabry-Pérot filters (FPF) were developed and are commercially available. The filters, based on bulk micromachining and $\mathrm{Si}-\mathrm{SiO}_{2}$ Bragg reflectors exhibit a high finesse up to 60 over the entire aperture of $\varnothing 1.9 \mathrm{~mm} .{ }^{[4]}$

However, the limitation to the MWIR spectral range is unsatisfactory, because many substances have strong and more specifiable absorption bands in the long-wave infrared (LWIR) from 8 to $12 \mu \mathrm{m}$ (fingerprint region). Therefore in recent years efforts have been made to expand the range to the LWIR. ${ }^{[5][6]} \mathrm{A}$ new dielectric material system is used for the reflectors. It is based on $\mathrm{Ge}$ and $\mathrm{ZnS}$, which is the state of the art in infrared interference filters, supplemented by a new low refractive material. The usage of all three components gives an additional degree of freedom to optimize reflectance, bandwidth, phase dispersion and - most important - to achieve stress compensation within the dielectric layer stack.

Unlike FTIR or grating based spectrometers, the usable tuning range of an FPF is normally limited to one FSR, which is the widest in first order operation. The second restriction is due to the limited bandwidth of the bragg reflectors. Last but not least, the mechanical displacement needed to tune the FP cavity through a wide spectral range increases. This increases the design complexity considerably, which is supplemental associated with the electrostatic actuation voltage, acceleration sensitivity and chip size.

The new DB-FPF presented in this paper, addresses these restrictions by dedicated design approaches: A dual-band reflector with two stop-bands in the designated ranges for the simultaneous usage of the first and second interference order was developed. A new electromechanical design with two springsuspended reflectors is under development, which significantly reduces actuation voltage and acceleration sensitivity. First results have been presented in [7] . 

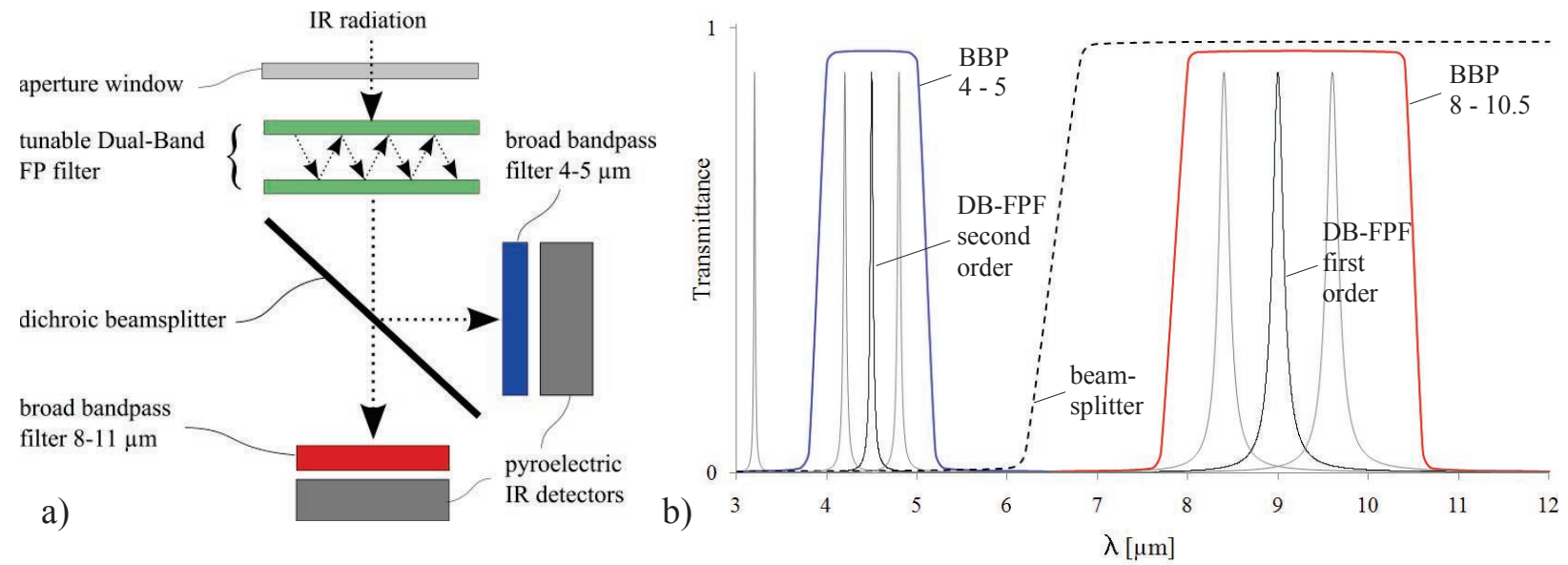

Fig. 1: Working principle of the dual-band FPF spectrometer module; a) optical layout b) spectral characteristics of the components (idealized): DB-FPF, broad bandpass filters and dichroic beamsplitter

The DB spectrometer module is based on a dual channel pyroelectric detector. The FPF passbands are separated by means of two broad bandpass filters and a dichroic beamsplitter, which exhibits high transmission in the LWIR and high reflectance in the MWIR. Compared to conventional 50:50 beamsplitters this solution significantly reduces signal losses. The working principle is shown in figure 1. To implement this in a compact detector configuration a true 3D integration of the optical and electrical components and the wiring is required. Figure 2 shows stages of the first prototypes in a TO8 package. The pyroelectric detectors have a specific detectivity of $D^{*} \geqslant 5 \times 10^{8} \mathrm{~cm} \sqrt{\mathrm{Hz}} / \mathrm{W}$.

One potential application, on which - amongst others - this work is directed to, is the monitoring of anesthesia gases. The majority of the mixture components can be most sensitively measured in the LWIR, whereas carbon dioxide $\left(\mathrm{CO}_{2}\right)$ and nitrous oxide $\left(\mathrm{N}_{2} \mathrm{O}\right)$ are usually measured between $4 \mu \mathrm{m}$ and $5 \mu \mathrm{m}$. On laboratory scale the spectrometer modules has been already successfully tested in the measurement of mixtures of anesthesia gases.
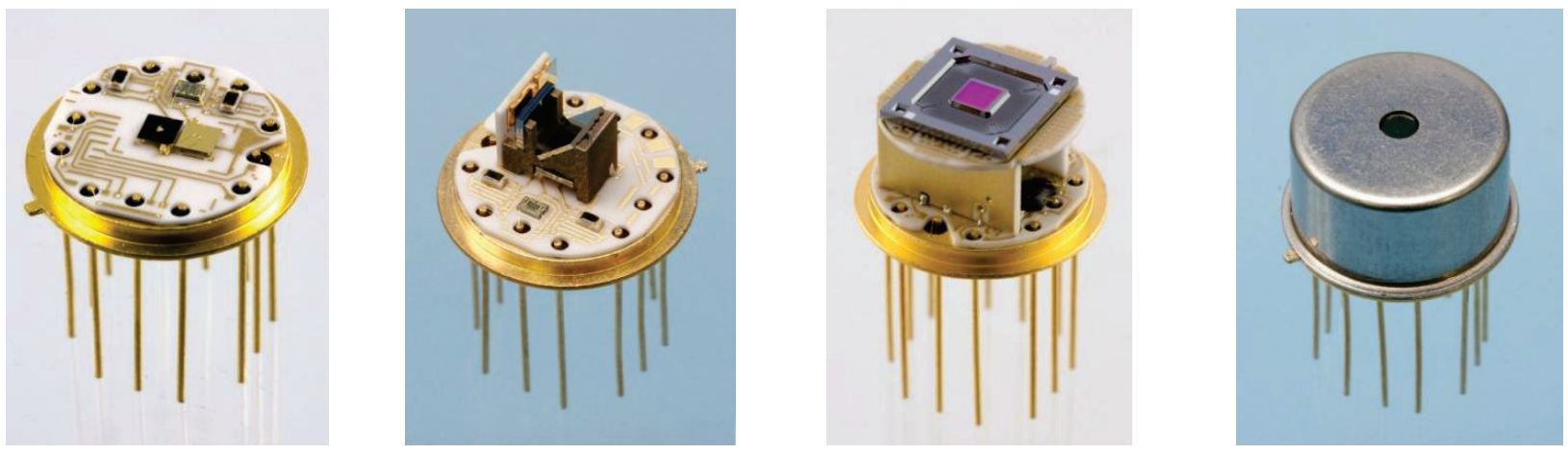

Fig. 2: Stages of the assembly of the Dual-band detector module

\section{Design considerations for a Dual-Band reflector and FPF}

The extinction coefficient of $\mathrm{SiO}_{2}$ is rising above $6 \mu \mathrm{m}$, so it cannot be used for reflectors beyond the MWIR. Alternatively a variety of high and low refractive index materials exists, which are established in conventional thin film interference filters. Ge and $\mathrm{ZnS}$ is a standard material system used for infrared interference filters. A new low refractive material has been introduced for the design of LWIR FP-filters. ${ }^{[6]}$ In contrast to $\mathrm{SiO}_{2}$ and poly-Si, the deposition of these materials are not standard processes in MEMS fabrication. Some work had to be done to integrate the reflector fabrication in the process flow. The layers are selectively deposited through a shadow mask utilizing an ion assisted deposition system (IAD). Therefore no additional structuring steps are necessary.

The maximum refractive index contrast $n_{H} / n_{L}$ for this material system is in the range of $2.5 \ldots 3$, which is a significant improvement compared to $\mathrm{Ge}-\mathrm{ZnS}\left(\mathrm{n}_{H} / \mathrm{n}_{L} \approx 1.8\right)$ and potentially results in simple designs with a limited number of layers and broad stop-bands.

Using dielectric stack mirrors, the dispersion of the reflectance phase shift $\phi$ cannot be neglected for low interference orders. Within the stop-bands $\phi$ can be normally approximated by a linear function of the wavenumber $\sigma$ with the slope $k_{\phi}(\sigma)$ at the design wavelength $\lambda_{\text {ref }}$ of the reflector. ${ }^{[8]}$ Reflectance phase 
dispersion results in two main effects on the performance of an FPF. Since $k_{\phi}(\sigma)$ is always negative the FSR and the width of the interference peak (measured as full width at half maximum, FWHM) are decreased by the same factor. Therefore the maximum usable tuning range is reduced, but on the other hand the spectral resolution slightly increases. As the other important effect the tuning sensitivity $\Delta \lambda / \Delta d$ decreases

$$
\Delta \lambda_{m}=\frac{2}{m}\left(1-\frac{k_{\phi}(\sigma)}{m \pi} \cdot \frac{1}{\lambda_{r e f}}\right)^{-1} \Delta d
$$

which is $2 / m$ for an ideal FPF without phase dispersion. This effect is highly undesirable for electrostatic actuation, because higher actuation voltages are the consequence. As a rule of thumb, the phase dispersion increases with the number of layers in the stack. A high refractive index contrast is therefore useful to reduce the influence of phase dispersion, because a less number of layers is needed to achieve a certain reflectivity. Further improvements are possible through an optimization of the layer sequence and thicknesses. ${ }^{[9]}$

Conventional bragg reflectors are based on a $\mid \mathrm{LH}$ l layer period, which produces a first stop-band around the design wavelength $\lambda_{\text {ref }}$, where $\mathrm{L}$ and $\mathrm{H}$ are of quarter wave optical thickness (QWOT), and higher order stop-bands for odd multiples $(3,5,7 \ldots$ QWOT). Nevertheless, such designs shall be referred to as single-band (SB) within this paper.

The new dual-band design approach with $|\mathrm{HLH}|$ or $|\mathrm{LHL}|$ basis periods results in a split stop-band symmetrical (in wavenumbers) to $\lambda_{\text {ref }}$. Figure 3 shows a comparison of two exemplary SB and DB designs. The tuning characteristic, that means the mechanical travel needed to spectrally tune the centre wavelength of the filter, is nearly identical in the first order for both designs.
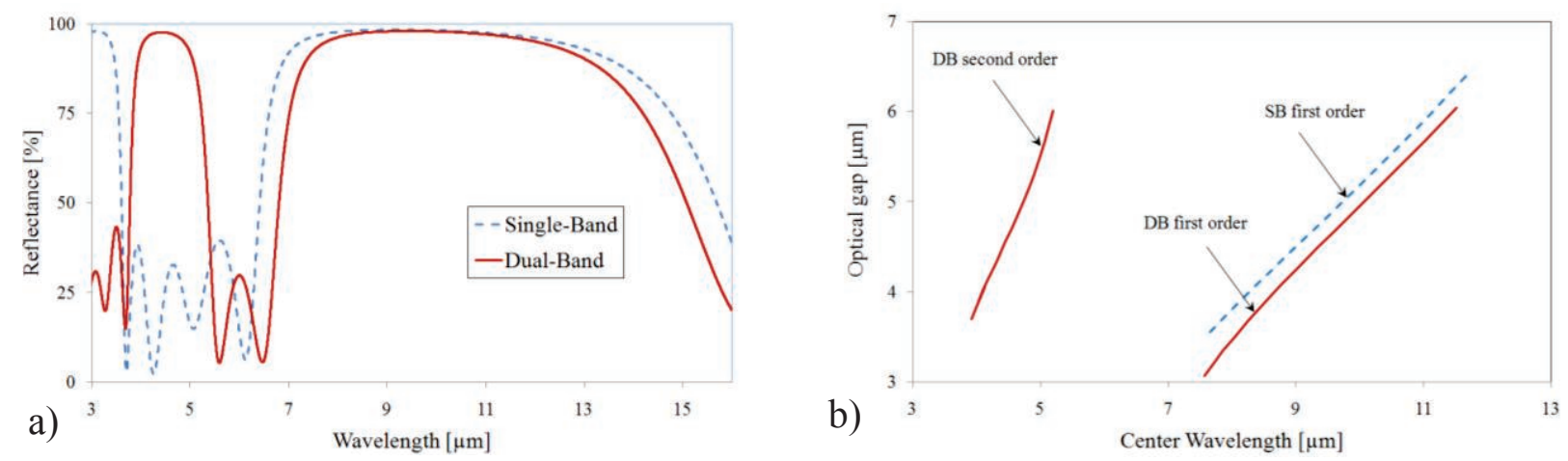

Fig. 3: Comparison of two exemplary reflector designs; a) reflectance spectra for a single-band $|\mathrm{LH}|^{2}$ stack $\left(\lambda_{\text {ref }}=9 \mu \mathrm{m}\right)$ and a dual-band $\left(\mathrm{H}-|\mathrm{HLH}|^{2}-\mathrm{H}\right)$ stack $\left.\left(\lambda_{\text {ref }}=6 \mu \mathrm{m}\right) ; \mathrm{b}\right)$ tuning characteristics for both reflectors

\section{Analysis and improvements of the reflector performance}

The performance of the real FPI always suffers from imperfections of the optical cavity, which is mainly caused by reflector curvature, tilting and surface roughness. These defects result in a certain distribution of the optical phase and therefore in a broadening and attenuation of the transmission peak. The cumulative effect on the finesse is usually described by the defect finesse $F_{D}$ or respectively by the effective finesse $F_{E}$

$$
\frac{1}{F_{E}^{2}}=\frac{1}{F_{R}^{2}}+\frac{1}{F_{D}^{2}}
$$

Equation 2 implies, that the contribution of each single defect to the effective finesse is independent from the others. Unfortunately this is not true for concurrently warped and tilted reflectors, because their corresponding phase distributions are correlated through the spatial distribution of cavity deviations within the filter aperture. Not only finesse but also peak transmittance and contrast are affected by surface defects. The peak attenuation depends on the ratio $F_{R} / F_{D}$. As a consequence it is not possible to compensate for defects by a higher reflectivity without a dramatic loss in transmission. In most practical cases $R=94 \ldots 97 \%$ are reasonable values, which gives a reflectivity finesse of $F_{R}=50 \ldots 100$. A very detailed examination of several kinds of defects has been given by Palik et. al. ${ }^{[10]}$.

A common approach to estimate the impact of macroscopic defects was described by Vaughan. ${ }^{[11]}$ The optical cavity is approximated by numerous cavity elements, each of them treated as an ideal FP interferometer but with an individual spacing. The effective transmittance spectrum of the entire aperture is then be calculated by summing the spectra of the elementary cavity elements. With this method one 
can determine the nature of the dominating defects from spectral transmission measurements (except microscopic defects like surface roughness).

Prior to this work, single-band FP-filters for the LWIR based on Ge-ZnS bragg reflectors and the new material system were developed and tested. ${ }^{[6]}$ With the standard Ge-ZnS system a very pronounced warping of more than $50 \mathrm{~nm}$ appears due to layer stress. At the same time a strong tilting effect was observed, which was mainly due to the early technological development stage but also caused by the asymmetrical chip layout. With the aid of the simulation method described above, the dominating defects could be identified and quantified. Figure 4 shows such an example, for which reflector tilting was determined to be about $220 \mathrm{~nm}$ and a spherical bowing of $60 \mathrm{~nm}$ (each reflector) was estimated. The asymmetrical shape of the peak is typical for concurrently warped and tilted reflectors.
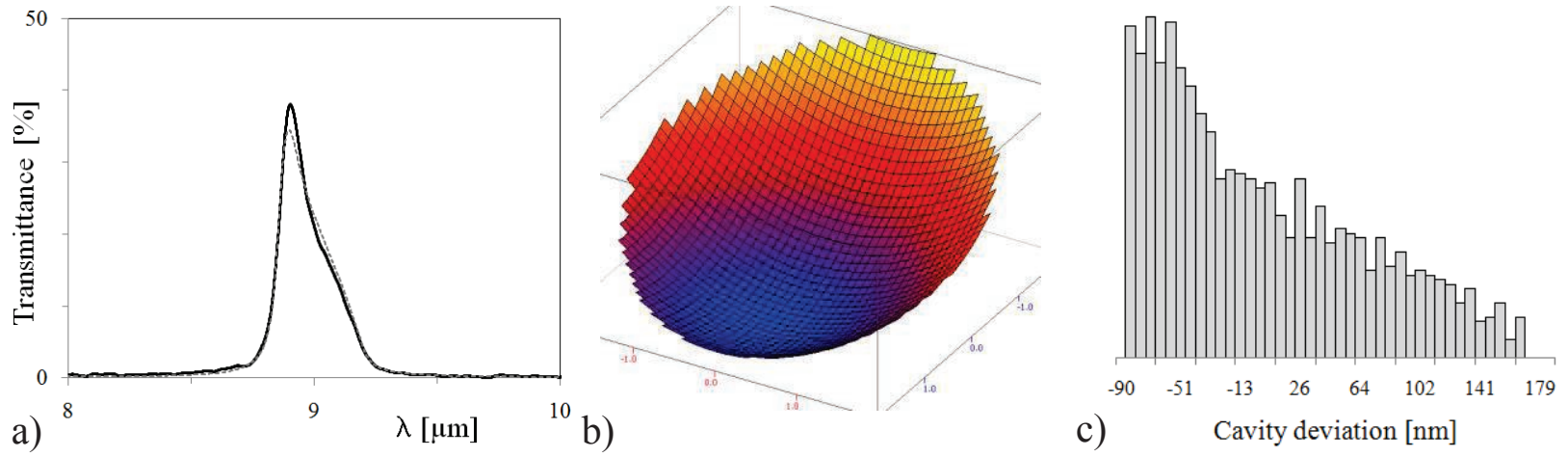

Fig. 4: Analysis and simulation of defects with the finite cavity method $(\approx 220 \mathrm{~nm}$ tilting, $60 \mathrm{~nm}$ peak-to-valley curvature); a) measured (solid curve) and simulated (dotted curve) transmittance spectra of a SB-FPF with a Ge-ZnS bragg reflector; b) estimated cavity profile; c) histogram of cavity deviations from the ideal value

Based on these findings the new DB reflector design and the deposition technology could be highly optimized and stress compensation with outstanding flat surfaces was achieved. The peak to valley curvature was found to be in the range of only $15 \mathrm{~nm}$, in some cases even down to $7 \mathrm{~nm}$ over the full aperture of $1.9 \mathrm{~mm}$. This corresponds to a defect finesse of at least 270 in the LWIR and 130 in the MWIR. The chip layout has been revised as well and is now completely symmetrical. Reflector tilting could be reduced to about $30-50 \mathrm{~nm}$, which results in a tilting finesse of better than 90 (LWIR) respectively 46 (MWIR). The surface roughness is less than $5 \mathrm{~nm}$, which is small enough to be neglected.
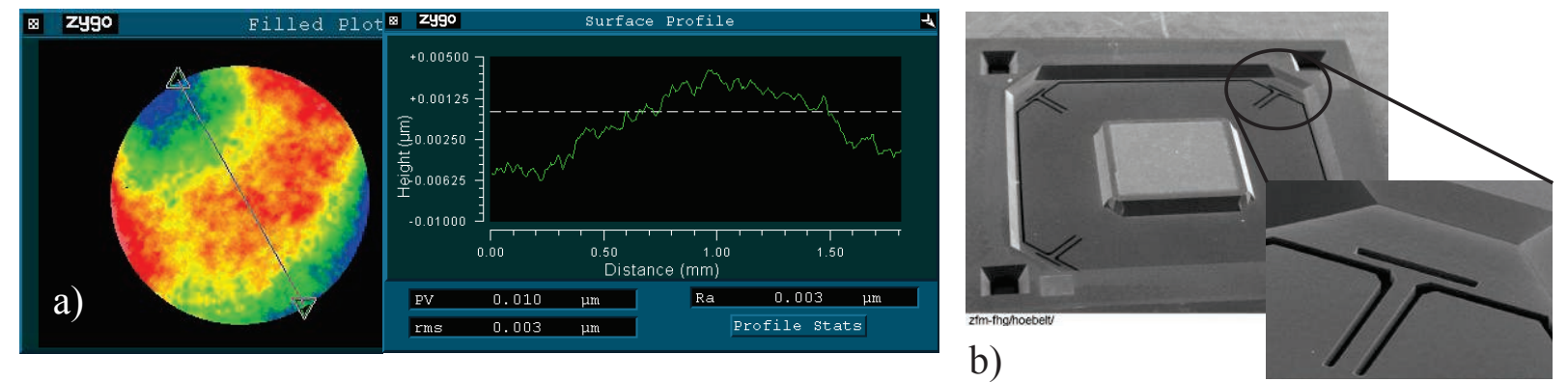

Fig. 5: a) Surface profile of a DB reflector measured with white light interferometer (WLI) 10nm b) SEM photograph of the complete chip and spring detail.

A tilting finesse of 46 in the MWIR band would degrade the filter transmission if the reflectivity finesse would be significantly higher. At the same time the spectral resolution in the second order is inherently higher compared to the first order (twice as high for an ideal FPF). For this reason it was decided to reduce the reflective finesse in the MWIR band to $\leq 35$. For the LWIR the reflectivity finesse was designed to be $\leq 70$. The reflectance phase dispersion is $1.24 \cdot 10^{-3} \mathrm{~cm}$ at $9 \mu \mathrm{m}$, which results in a mechanical travel of $1.8 \mu \mathrm{m}$ to tune the filter through $8-10.5 \mu \mathrm{m}$ and a tuning sensitivity $\Delta \lambda / \Delta d$ of 1.39 .

\section{Spectral characterization of dual-band reflectors and filters}

Reflectance measurements were performed with an FTIR spectrometer with an incidence angle of about $11^{\circ}$ and $4 \mathrm{~cm}^{-1}$ resolution. The spectra were corrected for the reflectance of the used gold reference $(R=98 \%)$. Figure 6a shows the good agreement between the reflector design and measured spectra.

First samples of fully functional DB-FPF have been fabricated, characterized and tested. Transmission measurements of the filters were performed with an FTIR spectrometer with an resolution of $4 \mathrm{~cm}^{-1}$. The half angle of the measurement beam was about $4^{\circ}$. Measured spectra are shown in figure $6 \mathrm{~b}$. 

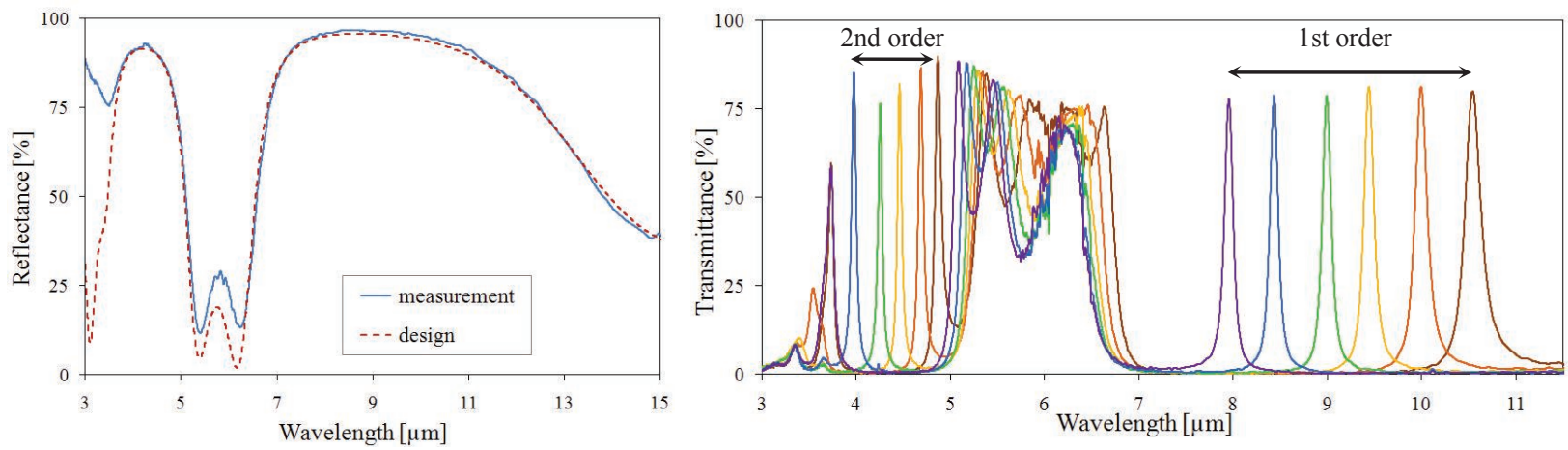

Fig. 6: Spectral characterization of DB reflectors and filters; a) DB reflector: comparison of simulated and measured spectral reflectance, b) Spectral transmittance spectra of the DB-FPF

The peak transmittance reaches high values of up to $81 \%$. An FWHM bandwidth of $200 \mathrm{~nm}$ down to $105 \mathrm{~nm}$ in the LWIR was achieved, which corresponds to a spectral resolution power $\lambda / \Delta \lambda$ of the filter of 50 up to 80 . In the second order (MWIR) the FWHM bandwidth was smaller than $80 \mathrm{~nm}$. The resulting spectral resolution power is 60 up to 80 , which is comparable to the LWIR. The actuation voltage needed for full tuning range was about 50 volts. Measurement results of the spectral performance are summarized in table 1.

Table 1: Spectral performance of the DB-FPF

\begin{tabular}{ccc}
\hline & second order & first order \\
\hline Spectral tuning range $[\mu \mathrm{m}]$ & $3.9-4.8$ & $8-10.5$ \\
Tuning sensitivity $\Delta \lambda / \Delta d$ & 0.6 & 1.39 \\
Control voltage $[\mathrm{V}]$ & 50 & 51 \\
FWHM [nm] & $55-80$ & $105-200$ \\
Peak transmittance [\%] & $75-90$ & $75-81$ \\
Reflectivity finesse & $\leq 35$ & $\leq 70$ \\
Effective finesse & $\leq 23$ & $\leq 56$ \\
Spectral resolution power $\lambda / \Delta \lambda$ & $60-80$ & $50-80$
\end{tabular}

\section{Improved MEMS design with reduced acceleration response}

The movable reflector carrier acts as a mass-spring-damper system and therefore exhibits acceleration sensitivity. The filter wavelength responds to external forces resulting from vibration, shock or turning in the gravitational field of the earth. The quasi-static acceleration response of the centre wavelength is $25 \mathrm{~nm} / \mathrm{g}$ up to $50 \mathrm{~nm} / \mathrm{g}$ for the conventional chip design with one spring suspended reflector.

A novel design approach with two spring-suspended reflector carriers was presented in [7], which significantly reduces the acceleration sensitivity. External forces, induced by gravitation or vibration, have nearly no influence on the optical cavity, if the mass and the spring stiffness of both reflector carriers are equal. The acceleration sensitivity depends only on the deviations of the mass or spring stiffness between both suspended parts. In addition, each reflector has to travel only half the distance. Both effects lead to significantly lower requirements on the control voltage respectively the electrode area. A schematic cross section of the chip design is shown in figure 7a. The reflector carriers are rectangular shaped and arranged perpendicular to each other. They are structured by wet-etching to create the electrode gaps and the optical cavity. The reflector carriers overlap each other in the middle and further with the chip frame of the opposite substrate. All these overlapping areas are used for electrostatic actuation as well as squeeze film dampers. First fabricated prototypes show a reduced gravitational influence of $\pm 4 \mathrm{~nm}$ in comparison to $\pm 28 \mathrm{~nm}$ for the design described above with one movable reflector only (fig. $7 \mathrm{~b}$ ). 

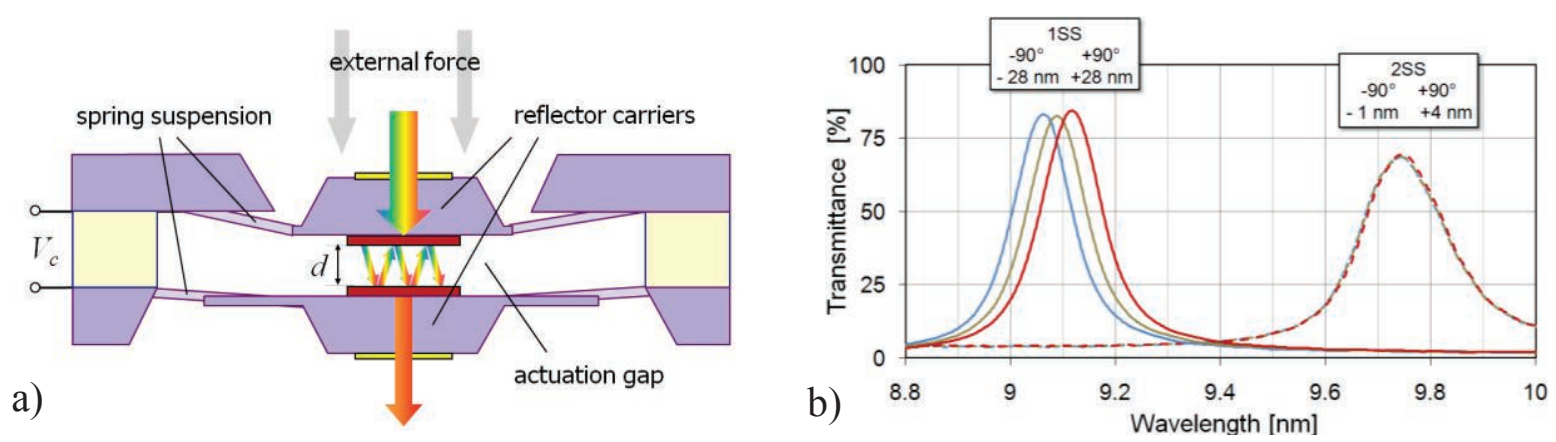

Fig. 7: a) Schematic cross-section; b) Comparison of measured spectra between one (1SS) and two (2SS) spring suspended reflectors under gravity influence

\section{Summary}

Based on an established and proven MEMS FPF design new long-wave and dual-band filters for simultaneous measurements in the MWIR and LWIR were developed and tested. A new set of three optical thin film materials is used for reflectors above $6 \mu \mathrm{m}$. The rigorous analysis of defects, a technological optimized and stress compensated reflector design and a revised chip layout were the basis for a high spectral performance. A finesse of up to 56 and transmission values of about $80 \%$ were achieved. A novel design approach, with two spring suspended reflectors, which dramatically reduces the acceleration sensitivity of the FP filter was successfully proven.

The authors would like to acknowledge the financial support by the German Federal Ministry of Education and Research, contract number 13N9489.

[1] Rossberg, D.: "Silicon micromachined infrared sensor with tunable wavelength selectivity for application in infrared spectroscopy", Sens. Actuators A 47 (1), 413-416 (1995)

[2] Noro, M., Suzuki, K., Kishi, N., Hara, H., Watanabe, T., Iwaoko, H., "CO2/H2O gas sensor using tunable Fabry-Perot filter with wide wavelength range", Proc. IEEE MEMS 2003 Conf., 319-322 (2003).

[3] Lehto, A., Orpana, M., Blomberg, M., "Electrically tunable fabry-perot interferometer produced ba surface micromechanical techniques for use in optical material analysis", European patent application EP0668490, (1995)

[4] Neumann, N.; Ebermann, M.; Kurth, S.; Hiller, K.: "Tunable infrared detector with integrated micromachined Fabry-Perot filter”. J.Micro/Nanolith. MEMS MOEMS 7(2), 021004-01 - 021004-9 (2008)

[5] Stupar, P. A.; Borwick, R. L.; DeNatale, J. F.; Kobrin, P. H.; Gunning, W. J.: "MEMS tunable FabryPerot filters with thick, two sided optical coatings", Proc. IEEE Transducers 2009 Conf., 1357-1360 (2009)

[6] Ebermann, M.; Neumann, N.; Hiller, K.; Gittler, E.; Kurth, S.: "Recent Advances in Expanding the Spectral Range of MEMS Fabry-Pérot Filters", Proc. SPIE, Vol. 7594, (2010)

[7] Meinig, M., Kurth, S., Hiller, K., Neumann, N., Ebermann, M., Gittler, E., Gessner, T.; "Tunable midinfrared filter based on Fabry-Pérot interferometer with two movable reflectors" Proc. SPIE, Vol. 7930, (2011)

[8] Mielke, S. L., Ryan, R. E., Hilgeman, T., Lesyna, L., Madonna, R. G., Van Nostrand, W. C., "Measurements of the phase shift on reflection for low-order infrared Fabry-Perot interferometer dielectric stack mirrors," Appl. Opt. 36, 8139-8144 (1997)

[9] Macleod, H. A.: "Thin-Film optical filters", IoP, Bristol and Philadelphia (2001)

[10] Palik, E. D., Boukari, H., Gammon, R. W., "Experimental study of the effect of surface defects on the finesse and contrast of a Fabry-Perot interferometer," Appl. Opt. 35, 38-50 (1996).

[11] Vaughan, J. M., "The Fabry-Perot Interferometer: History, Theory, Practice and Applications", Adam Hilger, Bristol (England), (1989) 